\title{
EUROPEAN PHYSICAL SOCIETY EPS Organized Conferences in 1985
}

\section{CONFERENCES}

18-22 March

5th EPS General Condensed Matter Physics Conference Berlin-West

Prof. J. Treusch, Institut für Physik der Universität

Prof. J. Treusch, Institut für Physik der
Postfach 500500, D-4600 Dortmund

\section{1-4 April}

7th European Conference on Surface Science (ECOSS 7)

Aix-en-Provence (F)

Prof. M. Gillet, Faculté des Sciences et Techniques de St-Jérồme

Rue Henri Poincaré 12, F-13397 Marseille Cedex 13

\section{5-19 April}

2nd European Conference on Atomic and Molecular Physics (ECAMP II)

\section{Amsterdam (NL)}

Prof. M.J. van der Wiel, FOM-Institute for Atomic and Molecular Physics,

Kruislaan 407. NL - $1098 \mathrm{SJ}$ Amsterdam

\section{1-5 July}

11th EPS Nuclear Physics Divisional Conference:

Intermediate Energy Nuclear Physics

with Electromagnetic Probes

Paris (F)

Dr. A. Gérard/Dr. S. Samour

CEN - Saclay - DPhN/HE, F-91191 Gif-sur-Yvette Cedex

\section{5-18 July}

17th Europhysical Conference on Macromolecular Physics:

Morphology of Polymers

\section{Prague (CS)}

Dr. Z. Pelzbauer, P.M.M. Secretariat, Inst. of Macromolecular Chemistry.

Dr. Z. Pelzbauer, P.M.M. Secretariat, Inst. of Macromolecular

CS -162 O6 Prague 6

18-24 July

International Europhysics Conference

on High Energy Physics

$$
\text { Bari (I) }
$$

Professor G. Preparata, Istituto di Fisica, via Amendola 173, 1-70 Bari

\section{2-6 September}

12th European Conference on Controlled Fusion and Plasma Physics

\section{Budapest $(\mathrm{H})$}

Dr. L. Pocs, Central Research Institute for Physics,

P.O. Box 49, H - 1525 Budapest

\section{2-6 September}

Trends in Quantum Electronics

Bucharest (R)

Dr. I.A. Dorobantu, Central Institute of Physics, P.O. Box 5222, R-Bucharest

\section{9-12 September}

15th European Solid State Device

Research Conference - ESSDERC

Aachen (D)

Dr. H. Henke, Institut für Halbleitertechnik, Rhein.-Westf- Technische

Hochschule, W Schottkihaus, Sommerfeldstrasse D-5100 Aachen

\section{7-26 September}

6th Summer School on Computing Techniques in Physics:

Software Engineering, Methods and Tools

in Computational Physics

Nové Mesto na Morave (CS)

Dr. J. Nadrchal, Institute of Physics CSAV.

Na Siovance 2, CS - 18040 Prague-Liben 8
ECA VOLUME*)

$9 \mathrm{~A}$

$9 \mathrm{C}$

9 B

$9 \mathrm{D}$

$9 \mathrm{E}$

no abstracts

booklet

9 F

$9 \mathrm{G}$

$9 \mathrm{H}$

no abstracts booklet
EPS DIVISIONS

Condensed Matter

Condensed Matter Surfaces and Interfaces

Atomic \& Molecular Physics

Nuclear Physics

Condensed Matter:

Macromolecular Physics

High Energy and Particle Physics
Quantum Electronics
Condensed Matter: Semiconductors and Insulators

Computational Physics Group 